\title{
Revisión y puesta al día en cáncer de lengua
}

\section{Review and updating in tongue cancer}

\section{García Kass AI*, Domínguez Gordillo AA**, García Núñez JA*, Cancela Rivas G***, Torres Salcines J***, Esparza Gómez GC*}

\section{RESUMEN}

Objetivos: Revisar y actualizar los aspectos epidemiológicos, factores de riesgo, diagnósticos y terapéuticos del cáncer de lengua.

Material y métodos: Se ha realizado una revisión de la literatura en la base de datos PUBMED, utilizando la palabra clave principal "cáncer de lengua" y los subtítulos (subheadings): "diagnóstico", "epidemiología”, "etiología", "genética", "mortalidad" y "tratamiento", sin restricciones en cuanto al tipo de estudio, seleccionando aquellos artículos considerados de interés para una revisión. La información se ha completado con otras fuentes bibliográficas como registros de cáncer y tratados de Oncología. Se ha restringido la búsqueda a artículos en inglés o castellano. Asimismo, se han utilizado los datos del Programa de Vigilancia, Epidemiología y Resultados Finales del Instituto Nacional del Cáncer (SEER) para obtener datos de incidencia y mortalidad de cáncer en los Estados Unidos, y de GLOBOCAN para los datos referentes a otros 184 países del mundo. Resultados: Se han revisado un total de 1200 artículos, con un periodo de búsqueda objeto de la revisión comprendido entre el 1 de enero de 1990 y el 31 de marzo de 2013, incluyéndose revisiones sobre la epidemiología del tumor, sobre los factores de riesgo genético, y sobre los distintos aspectos del diagnóstico y tratamiento, un estudio prospectivo y uno randomizado.

Palabras clave: Cáncer de lengua, diagnóstico, epidemiología, etiología, genética, mortalidad, tratamiento.

\section{SUMMARY}

Aim: To review and update the epidemiological aspects, risk factors, diagnostic and therapeutic aspects of tongue cancer.

Material and Methods: a review of the literature has been achieved in the PUBMED database, using the main keyword "tongue cancer", and the subheadings: "diagnosis", "epidemiology", "aetiology", "genetics", "mortality" and "treatment", without restrictions in terms of type of study, choosing only those papers considered of interest for a review. The information has been completed with other bibliographic sources such as cancer registries and Oncology essays. The research has been restricted to papers in English or Spanish. Additionally, data of the Surveillance, Epidemiology and End Results (SEER) Program of the National Cancer Institute have been used to obtain incidence and mortality data of cancer in the United States, and of GLOBOCAN for data concerning other 184 countries in the world.

Results: A total of 1200 papers have been reviewed, with a research period comprising between 1st January 1990 and 31st Mars 2013, including reviews about the epidemiology of the tumour, the genetic risk factors and the different aspects of the diagnosis and treatment, a prospective and a randomized study.

Key words: Tongue cancer, diagnosis, epidemiology, aetiology, genetics, mortality, treatment.

* Departamento de Estomatología III. Facultad de Odontología. Universidad Complutense de Madrid.

** Departamento de Medicina Preventiva y Salud Pública. Facultad de Odontología. Universidad Complutense de Madrid.

*** Hospital Central de la Defensa "Gómez Ulla". 
Fecha de recepción: 19 de junio de 2013.

Aceptado para publicación: 8 de julio de 2013.

García Kass AI, Domínguez Gordillo AA, García Núñez JA*, Cancela Rivas G, Torres Salcines J, Esparza Gómez GC. Revisión y puesta al día en cáncer de lengua. Av. Odontoestomatol 2013; 29 (5): 255-269.

\section{FRECUENCIA DE PRESENTACIÓN Y MORTALIDAD}

Los cánceres de la lengua, tanto de su parte móvil como de su base, son las neoplasias más comunes de la cavidad oral (si bien la base de la lengua pudiera considerarse como orofaringe). En EEUU, entre los casos diagnosticados de cáncer de lengua desde 2002 al 2006, se observó una incidencia de 2,8 por 100.000 habitantes/año, siendo el cambio porcentual anual de la incidencia de cáncer de lengua entre los años 1975 y 1999 de 0,4, y entre 1999 y 2006 de 2,0 . Esto quiere decir que en ambos periodos de tiempo se ha producido un incremento en la incidencia de la enfermedad cancerosa (1). Por otra parte, y teniendo en cuenta que se incluye, además de la parte móvil de la lengua, su base, entre los pacientes diagnosticados en 2002-2006 se encontró una tasa de mortalidad de 0,6 por 100.000 habitantes/año. En este caso, el cambio porcentual anual de la mortalidad de cáncer de lengua en los periodos 1975-1989, 1989-1999, y 1999-2006 es negativo, de $-1,6,-2,3$ y $-0,4$, respectivamente, lo cual implica una disminución de la misma (1). Basándose en las tasas de 2004-2006, el 0,29\% de los hombres y mujeres nacidos hoy serán diagnosticados de cáncer de lengua en algún momento de su vida (1). La estimación para 2011 en EEUU fue de 12.060 nuevos casos (8.560 hombres y 3.500 mujeres), y 2.030 fallecimientos (1.320 hombres y 710 mujeres) de cáncer de lengua (2).

En Europa, según datos de la IARC (Internacional Agency for Research on Cancer) la incidencia del cáncer de lengua (incluyendo base) entre los hombres oscila entre 0,8/100.000 hombres en Polonia hasta un máximo de 8/100.000 hombres en el Bajo Rin francés. En España, el mínimo lo vamos a encontrar en Albacete, con 2,1 por cada 100.000 hombres, frente al máximo de 5,2/100.000 en Asturias (3).

\section{HISTOPATOLOGÍA DE LOS TUMORES DE LENGUA}

\section{Lesiones y condiciones precancerosas}

Dentro de la clasificación de Speight (4), basada en criterios clínicos, podemos distinguir:

- Lesión precancerosa: Tejido morfológicamente alterado en el que es más probable que se desarrolle un cáncer que en los tejidos adyacentes aparentemente normales. Incluye leucoplasia, eritroplasia y eritroleucoplasia.

- Condición precancerosa: Estado generalizado asociado a un riesgo significativamente elevado de desarrollar cáncer. Incluye sífilis, fibrosis oral submucosa, queilitis actínica, disfagia sideropénica y liquen plano.

Cuando se acuñaron estos términos, se consideraba que el origen de una neoplasia en la boca de un paciente con una lesión precancerosa, se correspondería con el sitio del precáncer. Por otro lado, en el caso de las condiciones precancerosas, el cáncer podría aparecer en cualquier localización anatómica de boca o faringe. En la actualidad, se sabe que la mucosa de apariencia clínicamente "normal" en un paciente que presenta una lesión precancerosa, puede tener displasia, y es por este motivo por el cual Warnakulasuriya et al., en 2007, proponen unificar los conceptos de lesión precancerosa y condición precancerosa en uno solo: "desórdenes potencialmente malignos", para reflejar la amplia distribución anatómica de todas las presentaciones clínicas que tienen un riesgo de cáncer (5).

\section{Patología tumoral}

Los cánceres de la porción móvil de la lengua están situados por delante de la $\mathrm{V}$ lingual y corresponden 
a los dos tercios anteriores del órgano. Comprenden los bordes laterales, la cara dorsal y la cara ventral. Las neoplasias de la base de la lengua ocupan el tercio posterior de la lengua, por detrás de la $\mathrm{V}$ lingual. En algunos casos, la invasión tumoral masiva de la porción móvil de la lengua o de la totalidad de la lengua no permite determinar con certeza el lugar de origen del tumor.

Más del $90 \%$ de los tumores malignos de la lengua son carcinomas epidermoides. Los restantes incluyen carcinomas verrucosos y adenocarcinomas ocasionales que se originan en glándulas salivales menores de la lengua. Los sarcomas son raros; incluyen los que provienen de fibroblastos, músculo esquelético, vainas neurales y endotelio vascular, y también linfomas hodgkinianos y no hodgkinianos, que representan el espectro total de tejido conjuntivo, elementos neuroectodérmicos y tejido linfoide de los cuales deriva la lengua. Los tumores metastáticos de la lengua son raros y derivan principalmente de melanomas de la piel y carcinomas de mama y pulmón (6).

\section{Carcinoma de células escamosas (CCE)}

El carcinoma de células escamosas es un tumor maligno común a la piel, la boca y otras cavidades del cuerpo que están recubiertas por un epitelio escamoso o incluso cilíndrico. Esta neoplasia lingual es el carcinoma intrabucal de sitio único más común. Casi el $60 \%$ de las lesiones se originan en los dos tercios anteriores de la lengua y el resto proviene de la base. Hoy, se considera que el carcinoma del dorso de la lengua es una lesión rara y suele surgir de una anormalidad crónica de la mucosa, como glositis atrófica, alteración liquenoide o leucoplasia. En consecuencia, la mayor parte de los carcinomas de la lengua ocurren en los bordes de los dos tercios anteriores y la superficie ventral de la lengua. No siempre es posible separar con claridad esta última del suelo de la boca como sitio de origen de un tumor en particular. Es importante que el suelo de la boca sea el siguiente sitio más común de carcinoma bucal, lo que indica que la mucosa relativamente mal queratinizada de los lados y la superficie ventral de la lengua es más susceptible a transformación carcinomatosa. La mayor susceptibilidad de esta mucosa mal queratinizada resalta el efecto que tienen los agentes físicos y químicos locales (humo de tabaco, alcohol, traumatismo dental y xerostomía) en el desarrollo del cáncer bucal (6). En general, los tumores bien diferenciados son más comunes hacia las superficies anteriores de la lengua, aunque pueden encontrarse tumores anaplásicos y bien diferenciados en ambos sitios. Quizá por su localización y tendencia a ser menos diferenciados, como grupo, los carcinomas que surgen en el tercio posterior de la lengua, comparados con los de los dos tercios anteriores, son más grandes cuando se diagnostican inicialmente, es más frecuente que hayan invadido estructuras vecinas y más probable que se acompañen de fijación de la lengua (es decir, prueba de un tumor infiltrante avanzado). La frecuencia de metástasis a ganglios cervicales es directamente proporcional al tamaño y etapa del tumor primario de la lengua y, como cabría esperar, es más común (75\%) que los carcinomas más posteriores de la lengua presenten afectación ganglionar regional cuando se diagnostican por primera vez que los de los dos tercios anteriores (30\%) (6).

\section{ETIOPATOGENIA}

Es muy importante la capacidad para metabolizar carcinógenos o precarcinógenos, reparar el daño en el ADN, el control de las señales celulares y el ciclo celular. Tras la exposición a mutágenos, el carcinoma oral de células escamosas (COCE) puede aparecer si estos mecanismos son defectuosos. El COCE surge como consecuencia de múltiples eventos inducidos por los efectos de varios carcinógenos procedentes de hábitos tales como el tabaquismo, y es influido por factores ambientales, posiblemente virus en algunos casos, frente a una resistencia o susceptibilidad heredada. En consecuencia, el daño genético afecta a diversos cromosomas y genes, y es la acumulación de estos cambios la que conduce a carcinoma, en ocasiones a través de una lesión premaligna o potencialmente maligna clínicamente evidente (7). A pesar de que los factores de riesgo relacionados con el estilo de vida juegan un papel muy importante en la etiología, algunos pacientes parecen ser más susceptibles debido a un rasgo heredado en su dificultad para metabolizar carcinógenos o procarcinógenos, posiblemente junto con una difi- 
cultad para reparar el daño en el ADN. Las mutaciones pueden aparecer tras la exposición a agentes químicos (tales como asbestos), físicos (como las radiaciones ionizantes) y biológicos (microorganismos), y algunas pueden aparecer "espontáneamente" (7).

A nivel molecular, se produce un daño genético que se manifiesta a nivel celular con alteraciones en la señalización celular, el ciclo celular y/o en los mecanismos para reparar el daño celular o eliminar células no funcionales. La disminución o el aumento de la función de estos genes o sus productos está implicada en la carcinogénesis; los procesos de regulación celular se ven afectados a través de la transcripción de las proteínas implicadas (7).

La presencia de un campo con células genéticamente alteradas es un factor de riesgo de cáncer. El concepto de "cancerización de campo", introducido por primera vez por Slaughter et al., en 1953, se propuso para explicar el desarrollo de tumores primarios múltiples y recidivas locales de cáncer. Recientes hallazgos moleculares avalan el modelo de carcinogénesis en el que el desarrollo de un campo con células alteradas genéticamente juega un papel fundamental. En la fase inicial, una célula madre se altera genéticamente y forma un "parche", una unidad clonal de células hijas alteradas (8). El siguiente paso en la carcinogénesis epitelial es la transformación del parche en un campo en expansión. Alteraciones genéticas adicionales son necesarias para que esto se produzca y, finalmente, este campo acaba desplazando a la mucosa normal. En la mucosa de cabeza y cuello, se han detectado dichos campos con dimensiones de más de $7 \mathrm{~cm}$ de diámetro, que normalmente no son detectados mediante las técnicas diagnósticas de rutina. Finalmente, la divergencia clonal permite el desarrollo de uno o más tumores dentro de un campo de células preneoplásicas. Una implicación clínica importante es que estos campos normalmente permanecen tras la cirugía del tumor primario y pueden dar lugar a nuevos cánceres, denominados por los clínicos como "segundo tumor primario" o "recurrencia local". De esta forma, el diagnóstico y tratamiento de los cánceres epiteliales no debería centrarse únicamente en el tumor, sino también en el campo a partir del cual se desarrolló (8).

\section{FACTORES DE RIESGO}

\section{Tabaco}

El tabaco es el principal factor de riesgo asociado al desarrollo de lesiones premalignas y cáncer oral. 8 de cada 10 pacientes con cáncer oral son fumadores de tabaco en sus diversas formas: cigarrillos, puros, tabaco de mascar, tabaco en pipa. En el humo inspirado del tabaco existen más de 30 sustancias carcinogénicas conocidas. Las más importantes son los hidrocarburos aromáticos policíclicos y las nitrosaminas, que están fuertemente asociadas al cáncer de la cavidad oral y laringe-faringe, y en particular, al cáncer de lengua, siendo la relación directamente proporcional a la cantidad consumida. El riesgo relativo es de 1,52 en fumadores de una cajetilla/ día, aumentando a 2,43 con 2 cajetillas/día. Se ha demostrado que la interrupción del tabaquismo disminuye el riesgo de aparición de lesiones premalignas y malignas. Tanto es así que, a los 15 años de haber cesado el hábito de fumar, el riesgo de padecer displasia de las mucosas es similar a los que nunca han fumado. El riesgo de cáncer de cavidad oral disminuye un $30 \%$ cuando transcurren entre 1 y 9 años de haber dejado de fumar y un 50\% a partir de 9 años (9). En los países occidentales, fumar cigarrillos, puros o pipa es el hábito más común, pero en el sudeste asiático, especialmente en la India, fumar bidi (un tipo de tabaco barato) está ampliamente extendido. En algunas regiones, fabrican sus cigarros y los fuman a menudo colocando el extremo encendido del cigarro en el interior de la boca. Esta modalidad de fumar cigarrillos la encontramos en las Islas del Caribe, Colombia, Panamá, Venezuela, Cerdeña, Filipinas y la India. La relación existente entre fumar invertido y el cáncer de paladar está bien establecida en la India (10). Respecto a los diferentes hábitos de fumar tabaco, la OMS establece que fumar cigarros o puros incrementa el riesgo de sufrir cáncer oral en 6 veces, mientras que el fumar bidi lo hace en 36 veces. Aproximadamente el $8 \%$ de todos los casos de cáncer oral pueden ser atribuidos únicamente al tabaco (10). Respecto a los fumadores de marihuana, hay que precisar que el humo de la marihuana tiene 4 veces más alquitrán y $50 \%$ más de concentración de benzopirenos e hidrocarburos aromáticos que el humo del tabaco; sin embargo, es difícil demostrar que el cannabis sea un factor 
de riesgo independiente, ya que la gran mayoría de los consumidores de marihuana son fumadores y consumidores de alcohol. En un estudio que incluyó 173 pacientes con carcinoma de células escamosas y 176 controles sanos, cuando se ajustó el riesgo por tabaquismo y alcoholismo se encontró que el riesgo para padecer cáncer era 2,6 asociado al consumo de marihuana en forma proporcional a la cantidad (11).

\section{Alcohol}

El etanol puro, por sí mismo, no es una sustancia carcinogénica. Sin embargo, se asocia a sustancias carcinógenas que actúan como desencadenantes de la acción tóxica del alcohol. Así, el alcohol ejercería un efecto cáustico aumentando la permeabilidad de la mucosa oral y permitiendo el paso de otros carcinógenos como el tabaco. El riesgo de cáncer de orofaringe en bebedores es 6 veces mayor que en no bebedores y el riesgo de muerte por cáncer de orofaringe es 4 veces mayor en los alcohólicos (12). La dosis tiene una relación directamente proporcional con la posibilidad de tener un cáncer de cavidad oral; después de ajustar la variable tabaquismo, el riesgo relativo de cáncer de orofaringe se incrementa de 1,0 en los consumidores de $<25 \mathrm{~g} /$ día de alcohol a 125 en los que consumen $>100 \mathrm{~g} /$ día (13). Los carcinógenos y su cantidad varían de acuerdo al tipo de bebida alcohólica. La cerveza contiene nitrosodimetilamina y el vino y destilados diferentes tipos de taninos. Cuando se compara el tipo de alcohol con el tipo y cantidad de carcinógenos se observa que los "licores oscuros" como whisky, ron añejo y coñac contienen una mayor proporción de los carcinógenos éster y acetaldehído que los licores claros (vodka, ginebra, ron claro); la proporción de cáncer orofaríngeo y de laringe supraglótica es mayor en los consumidores de alcoholes oscuros; los consumidores de vino y cerveza tienen mayor proporción de cáncer de la cavidad oral (14).

El sinergismo de la asociación alcohol-tabaco en la génesis del carcinoma epidermoide de cavidad oral ha quedado demostrado. Esta asociación incrementa el riesgo en un $50 \%$ comparado con la población no expuesta a la intoxicación; el riesgo de un no bebedor que fuma 40 cigarrillos al día se multiplica por 2,5, al igual que en un bebedor excesivo que no fuma; sin embargo en un fumador bebedor el riesgo relativo se incrementa a $16(12,15)$. Con respecto a los colutorios con alcohol, los estudios que tratan de relacionar cáncer oral y colutorios son inconsistentes y contradictorios (16).

\section{Micosis}

No está claro si las levaduras, generalmente cándidas, son elementos sobreinfectantes de la lesión o agentes específicos productores de la misma. La prevalencia de infección por Candida (habitualmente albicans) es mayor en todas las enfermedades epiteliales intrabucales, pero más frecuente en afecciones malignas de la boca que en lesiones no malignas (6).

\section{Deficiencias nutricionales}

La condición nutricional más importante asociada al cáncer oral en los países occidentales es la disfagia ferropénica. Otro subtipo de ferropenia sin disfagia se ha encontrado entre los pacientes con cáncer oral sin hábito tabáquico ni enólico. Incluso en estudios intermedios de déficit de hierro se encuentra una atrofia de la mucosa, que asociada a otros factores de riesgo puede incrementar la actividad mitótica y disminuir la capacidad de reparación del epitelio (10). Pacientes con déficit de vitamina A han sido considerados de alto riesgo de transformación maligna de la mucosa de cavidad oral. El papel de la vitamina A es controlar la diferenciación celular. El déficit de esta vitamina puede desencadenar alteraciones celulares similares a aquellas inducidas por carcinógenos químicos. De todos modos, no hay una evidencia firme de que el déficit de vitamina A actúe en la patogénesis del cáncer oral. Además de la vitamina $\mathrm{A}$, la $\mathrm{C}$ y algunos elementos, como el cinc, se han asociado con la patogénesis del cáncer oral tanto en hombres como en animales (10).

\section{Infecciones víricas}

El primer estudio que buscó una relación entre COCE y virus herpes simple (VHS) sugirió una relación en- 
tre ambos, pero a pesar de que se han encontrado resultados interesantes, aún no se puede hablar de una relación causal entre VHS y COCE (17).

Una variante anaplásica del carcinoma epidermoide, denominada linfoepitelioma (por la combinación de células linfoides y epiteliales malignas mal diferenciadas) o carcinoma nasofaríngeo, ocurre en el tercio posterior de la lengua, aunque con menor frecuencia de lo que se presenta en cualquier otra parte de la bucofaringe (anillo de Waldeyer) y en la nasofaringe. En estos sitios, se ha establecido una asociación epidemiológica entre el virus Epstein-Barr (VEB) y este tumor, no quedando demostrado el papel del virus (6).

El virus del papiloma humano (VPH) es un virus epiteliotropo del que se han identificado más de 100 genotipos, algunos de los cuales, como VPH-6 y VPH11 típicamente se asocian con lesiones benignas como verrugas y papilomas, y por ello se denominan benignos, mientras otros como VPH-16 y VPH-18 se asocian fuertemente con lesiones malignas, y por ello reciben el nombre de genotipos malignos, oncogénicos o de alto riesgo (17). La evidencia de la infección por VPH, junto con una relación clonal entre el VPH y el tumor, tal y como se ha mostrado por la integración viral en el genoma de las células huésped, podría sugerir un papel causal en la carcinogénesis, y no ser meramente un invasor secundario. La identificación de los genes transformantes del VPH como E6 y E7, antígenos de cápside de VPH, en el COCE refuerza el papel oncogénico para el VPH. Además, estudios serológicos han confirmado que $\mathrm{VPH}-16$ es un factor de riesgo para COCE, ya que se han encontrado anticuerpos frente a E6 y E7 (considerados como indicadores de tumores transformados por la invasión de VPH-16) en carcinomas orofaríngeos (17). La tasa de detección del VPH en COCE en diversos estudios oscila entre 0 y 94\%. La detección del virus es mayor cuando el análisis se realiza mediante hibridación in situ y reacción en cadena de la polimerasa (PCR), y los estudios que han utilizado estas técnicas han identificado las secuencias de VPH 11, 16 o 18 hasta en un $60 \%$ de los COCE y un $40 \%$ de las lesiones orales potencialmente malignas (17-19). Además, VPH es identificado en un número significativamente mayor de pacientes con COCE que en controles. Estudios recientes han implicado al VPH particularmente en un subtipo de tumor pobremente diferenciado con características histológicas basaloides que aparecía en la parte posterior de la lengua y en las fauces. Un cuarto de los CCE de cabeza y cuello contuvieron $\mathrm{VPH}$, y de éstos, 90\% fueron VPH-16. Alrededor del $57 \%$ de los tumores de amígdalas fueron positivos para VPH, en comparación con $12 \%$ de los tumores intraorales (20). Gillison et al. mostraron que los cánceres orofaríngeos VPH-positivos aparecieron principalmente en amígdala palatina/lingual, tenían menos probabilidades de aparecer en pacientes consumidores moderados/severos de tabaco y alcohol, presentaban predominantemente morfología basaloide y menos mutaciones de p53, y tenían una mayor supervivencia en comparación con los VPH-negativos (21). Kantola et al. mostraron que ninguno de sus 105 tumores de la parte móvil de la lengua, incluyendo tumores en distintos estadios, fueron positivos para VPH (22). Los datos del estudio de Liang et al. sugieren que la incidencia del VPH en el cáncer de la parte oral de la lengua es baja y es poco probable que este virus juegue un papel importante en la etiología, patogenia y resultados clínicos en el cáncer de la parte oral de la lengua (23), mientras que los datos de Dahlgren et al. sugieren que la presencia de VPH es un factor pronóstico favorable en los cánceres de base de lengua (24). El VPH se transmite mediante contacto directo; principalmente por relaciones sexuales (vaginales, orales bucales), así como la por transmisión digital y a través de superficies epiteliales maceradas. Si bien no se han realizado estudios epidemiológicos que evalúen la transmisión oral directa del VPH, los datos disponibles relacionan la infección oral con el sexo oral y el contacto bucal directo $(25,26)$.

\section{Sífilis}

En cuanto a la sífilis, en el pasado existió un riesgo mayor de tres a uno de cáncer de lengua en pacientes con esta infección confirmada serológicamente, y había una frecuencia mayor de sífilis en enfermos con cáncer bucal que en pacientes con leucoplasia de la boca o tumores malignos en cualquier otra parte del cuerpo. Esta frecuencia mayor de carcinoma de la lengua se relacionó con la ocurrencia de glositis atrófica como una manifestación tardía de sífilis y los carcinomas linguales relacionados se localizaban en el dorso. Desde esa época, la sífilis ter- 
ciaria, la glositis atrófica luética y el carcinoma de la lengua se han hecho muy raros y es posible que la desaparición virtual del carcinoma de este tipo explique el incremento observado de carcinoma de los dos tercios anteriores en mujeres (6).

\section{DIAGNÓSTICO}

El carcinoma de la lengua normalmente se presenta clínicamente como una úlcera, una masa exofítica o una infiltración profunda con grados de induración variables. Muchas veces, estas lesiones son indoloras, lo que podría explicar el diagnóstico tardío de las lesiones del tercio posterior (27). Su naturaleza silente puede también resultar en presentación con nódulos cervicales asintomáticos. Los pacientes raramente presentan disfagia o dificultad en el habla. El diagnóstico precoz del COCE requiere un elevado índice de sospecha clínica. Las lesiones malignas tempranas pueden pasar desapercibidas debido a la incapacidad del clínico de detectar posibles cambios anatómicos. Las lesiones sintomáticas son más fáciles de detectar debido a las molestias referidas por el paciente: dolor lingual, bulto en la lengua, dolor de oídos, sangrado, masa en el cuello, cambios en la voz, disfagia. Los síntomas se asocian con más frecuencia a las lesiones de la lengua móvil que a las de la base de la lengua 28). La combinación de colorantes con técnicas de imagen han contribuido de un modo importante, entre otras cosas a la localización del ganglio centinela (29). Es deseable, en el preoperatorio, conocer de forma precisa la extensión del carcinoma de lengua con el fin de planificar tanto la resección como la reconstrucción, y para dar al paciente información acerca de la posible limitación funcional y el pronóstico. El asesoramiento clínico de la extensión, especialmente en las lesiones del tercio posterior de la lengua, normalmente es difícil e impreciso (27). El escáner mediante tomografía computarizada (TC) es ampliamente utilizado como una ayuda para determinar en el preoperatorio el estadio de los ganglios del cuello y para evaluar la lesión primaria. Se ha convertido en el método estándar frente a otras técnicas de imagen. Para evaluar las metástasis regionales, se encontraron tasas de error de 7,5-28\%, en comparación con tasas de $16 \%$ en el caso de la resonancia magnética y del 20-28\% para el examen clínico (30). Las nuevas técnicas de tomografía computarizada por emisión de fotón único (SPECT) y tomografía por emisión de positrones (PET) aún no están muy extendidas. En estudios preliminares, se ha observado que el PET con fluodesoxiglucosa F18 podría ser útil para identificar recurrencias y enfermedad oculta (30), mientras que en el caso del SPECT aún se debe determinar su papel a la hora de establecer diagnóstico por imagen en cabeza y cuello (31).

\section{FACTORES PRONÓSTICO}

El curso clínico de un paciente con COCE está determinado por las características específicas del tumor primario, las características del huésped y el tratamiento aplicado. Entre las características específicas del tumor, se han estimado tanto factores clínicos como histológicos como posibles factores pronóstico (32). A pesar de que el manejo del COCE depende notablemente del sistema de estadificación TNM, que se basa en la evaluación clínica, el estadio no siempre es suficiente para determinar el pronóstico. Aunque la mayoría de autores aceptan que el estadio de la enfermedad tiene una influencia crucial en el curso de la enfermedad, concretamente el tamaño tumoral que se ha asociado con un riesgo incrementado de recidiva local y supervivencia pobre, algunos tumores pequeños T1 se comportan de una forma agresiva y presentan de forma poco predecible un pronóstico pobre (22). El estado de los ganglios linfáticos cervicales es el factor pronóstico más importante para el COCE, ya que el desarrollo de metástasis cervicales reduce la supervivencia a 5 años en un 50\% (33). El patrón de diseminación del carcinoma metastático de lengua es drenar en primer lugar a los ganglios linfáticos cervicales. Las metástasis cervicales son frecuentes y se ha descrito su presencia en hasta un 30\% de "cuellos negativos" clínicos (34). De hecho, las metástasis tardías a los ganglios linfáticos cervicales tras el tratamiento inicial son el principal problema en el manejo de los carcinomas tempranos de lengua (estadios I y II), debido a la elevada incidencia de metástasis ocultas (35). El pronóstico en este grupo de pacientes es pobre, razón por la cual algunos clínicos son partidarios de tratar el cuello clínicamente negativo mediante disección selectiva de cuello o radioterapia (36). Las metástasis a distancia pueden ocurrir si se 
ha perdido el control locorregional de la enfermedad. A pesar de que el carcinoma de la lengua normalmente permanece clínicamente confinado a la cabeza y cuello hasta estadios tardíos de la enfermedad, las necropsias han demostrado que, aunque la lesión primaria se cure en prácticamente la mitad de los casos, las metástasis ganglionares regionales estaban presentes en el momento de la muerte en un $71 \%$ de los casos, y las metástasis ganglionares a distancia y las hematógenas estaban presentes en un $35,4 \%$ y $58,5 \%$, respectivamente. La localización más frecuente para la diseminación hematógena fue el pulmón (37).

Desde el punto de vista clínico, los cánceres de la base de la lengua son insidiosos y su pronóstico es malo. Estos cánceres pueden crecer según un patrón infiltrante o exofítico. A causa de que la base de la lengua carece de fibras de dolor, estos tumores suelen ser asintomáticos hasta que ya han evolucionado de manera significativa (38). Las metástasis a ganglios linfáticos son comunes debido al abundante drenaje linfático de la base de la lengua. De hecho, el $75 \%$ aproximadamente de pacientes con cáncer de base de lengua tienen ganglios cervicales positivos a su ingreso; el $30 \%$ de casos serán bilaterales. En un $22 \%$, habrá afectación histológica ganglionar en casos clínicamente negativos. Raras veces se diagnostican lesiones asintomáticas, sobre todo en base de lengua que se visualiza solo mediante examen con espejo indirecto y el síntoma más precoz es una leve odinofagia (39). Los ganglios linfáticos cervicales que suelen verse complicados son los de los niveles II y III. El desarrollo de segundos tumores primarios tiene un impacto negativo en el pronóstico de los COCE de cabeza y cuello (40).

Entre los factores histológicos, los patólogos han destacado el grado de queratinización, pleomorfismo nuclear, frecuencia de mitosis, reacción inflamatoria, así como invasión vascular, naturaleza de la invasión y estado de los márgenes quirúrgicos (32). En la actualidad, la mayor parte de los autores reconocen que el sistema de Broders y de la OMS de gradación histopatológica de malignidad en el carcinoma epidermoide de cabeza y cuello poseen escaso valor predictivo, debido a la naturaleza subjetiva de la medición basada en pequeñas biopsias de tumores histológicamente heterogéneos (41).

\section{TRATAMIENTO}

\section{Cirugía}

La cirugía es, a menudo, el tratamiento primario de los tumores T1 y T2 de los dos tercios anteriores de la lengua, y se combina con radioterapia en las lesiones de mayor tamaño, de localización más posterior y de histología más agresiva. Las pequeñas lesiones de la zona anterior de la lengua probablemente funcionan mejor sólo con cirugía (42). El objetivo de la cirugía es conseguir la escisión del carcinoma con un margen de tejido normal $(2 \mathrm{~cm})$, pero esto en la lengua puede resultar difícil, ya que el tumor puede infiltrar profundamente o tener un margen mal definido con islas de células tumorales, dificultando precisar la localización del margen de resección. Además, la lengua se puede comparar con cualquier otro compartimento fascial del cuerpo en el que no existe septum que restrinja la diseminación tumoral (27). En el carcinoma de lengua móvil, el riesgo de metástasis regional está significativamente asociado con la profundidad de la invasión tumoral. Los patrones de diseminación del cáncer a los ganglios linfáticos son predecibles, basados en la localización anatómica del tumor primario. De esta forma, en ausencia de evidencia clínica de afectación cervical, las características patológicas del tumor primario junto con su sitio de origen y estadio $\mathrm{T}$ clínico se usan para valorar el riesgo de metástasis regionales y, por tanto, de la necesidad de una disección de cuello (43). La cirugía normalmente presenta un pobre resultado si existe diseminación regional resultando frecuentemente en disección de cuello tardía (34), siendo el cuello ipsilateral el sitio más frecuente de fracaso de la resección local. La disección radical de cuello se consideraba tradicionalmente como la modalidad terapéutica de elección en caso de evidencia clínica de metástasis cervicales. Mediante esta disección se retiran los grupos ganglionares I, II, III, IV, V sin preservar la vena yugular interna, ni el músculo esternocleidomastoideo ni el nervio accesorio del espinal. Sin embargo, el sacrificio de estas estructuras vitales produce una marcada reducción de la calidad de vida de los pacientes. Los avances en cuanto a conocimientos de anatomía del cuello, una mejor comprensión del comportamiento biológico de los tumores y el desarrollo de mejores técnicas quirúrgicas han contribuido a la emergencia de la disección funcional de cuello, con excelentes 
resultados en cuanto a supervivencia. Se realiza cuando los ganglios linfáticos comprometidos no presentan invasión extracapsular, respetando las estructuras periganglionares. Mediante este tipo de disección se preservan las estructuras no linfáticas, disminuyendo la morbilidad y mejorando la recuperación postoperatoria del paciente. Así, la disección radical de cuello se reservaría a los pacientes con evidencia clínica o radiológica de afectación ganglionar, cuando la eliminación completa del tumor es inalcanzable sin el sacrificio de los nervios craneales, la vena yugular interna y el músculo esternocleidomastoideo (43). En pacientes con cuello negativo al examen clínico (N0) y riesgo de metástasis linfáticas tempranas está indicada la disección selectiva de cuello (no solo como tratamiento sino para evaluar la necesidad de tratamiento adicional). Consiste en retirar uno o más grupos linfáticos con posible compromiso metastático. En el caso de los tumores de la cavidad oral, el tipo de disección selectiva a realizar es la supraomohioidea, que eliminaría la mayor parte de los nódulos linfáticos que podrían estar implicados en metástasis ocultas de la lengua (posiblemente hasta un 70\%). Se retiran los grupos ganglionares I, II y III del cuello, resultando en una menor morbilidad y un efecto menos profundo en el drenaje linfático que la disección radical (34). Dadas las dificultades que presenta la escisión quirúrgica y la elevada incidencia de metástasis regionales, la mayor parte de autores están de acuerdo en dar radioterapia postoperatoria a los pacientes con márgenes quirúrgicos involucrados, dos o más metástasis ganglionares, y aquellos con evidencia de diseminación extraganglionar (44). La glosectomía total se realiza en carcinomas avanzados que incluyen base de la lengua. Tienen asociada una elevada mortalidad y morbilidad (45). Existe un alto índice de fracaso a la hora de conseguir márgenes quirúrgicos claros. La mayor parte de los pacientes consiguen recuperar un adecuado, pero dificultoso habla, pero el principal problema sigue siendo tragar sin aspirar, y muchos pacientes requieren la laringotomía para evitar este problema. El pronóstico a largo plazo tras la glosectomía total es pobre, siendo muy penosa la recuperación (46).

\section{Radioterapia}

La radioterapia consiste en dirigir rayos de alta frecuencia a una zona cuyo tamaño y volumen se de- terminan por adelantado. Es, por tanto, un tratamiento local que hace posible la destrucción selectiva de las células cancerosas, mediante la ruptura de sus cromosomas principalmente. En los tumores que se conocen como radiosensibles, las células cancerosas tienen una sensibilidad mayor a la radiación (mayor vulnerabilidad a la misma) que las células normales que las rodean, lo cual hace posible matar a dichas células cancerosas sin dañar las células sanas que rodean al tumor. La radioterapia se puede aplicar mediante un rayo externo (teleterapia), mediante un implante radiactivo (braquiterapia) o mediante una combinación de ambas.

A) La teleterapia, en la que desde un equipo emisor se producen radiaciones que atraviesan los tejidos englobando un volumen de tratamiento previamente definido sobre un soporte de imagen por un sistema informático. Estos equipos son normalmente aceleradores lineales que emiten fotones de alta energía (normalmente entre 6 y $23 \mathrm{MV}$ ), o electrones; y más raramente, bombas de cobalto-60 que emiten radiación monoenergética de 1,02 MV.

La radioterapia con rayo externo utilizado para tratar carcinoma de la lengua, claramente resulta en la irradiación de una extensa cantidad de tejido colindante, resultando a menudo en complicaciones a corto plazo como mucositis o infección por Candida, además de las complicaciones a largo plazo de xerostomía, pérdida del gusto, y ocasionalmente osteorradionecrosis (47).

B) La braquiterapia, en la que se utilizan isótopos radioactivos (el más común el iridio-192) que mediante técnicas quirúrgicas sencillas se colocan en el interior o en contacto con el tumor que deseamos tratar. Se trata de endobraquiterapia por cuerpos radioactivos en fuentes selladas. El primer cuerpo radioactivo utilizado fue el radio226; su interés reside en su largo período de desintegración (1.620 años), ya que deja de ser necesario renovar el material. Sin embargo, pueden producirse fugas de radón, un gas eminentemente tóxico. La elevada energía de los fotones hace imposible una protección radiológica eficaz por pantalla de plomo. El radio se utiliza en forma de agujas rígidas, relativamente voluminosas. El cuerpo radiactivo no se reparte en toda la longitud de la aguja. 
Las localizaciones anatómicas susceptibles de ser tratadas con braquiterapia incluyen: el labio, suelo de boca, lengua móvil, base de lengua, mucosa yugal, región amigdalina, nasofaringe, base de cráneo y ganglios cervicales. La ventaja de la braquiterapia es la aplicación de una alta dosis de radiación al tumor minimizando a la vez la dosis en los tejidos adyacentes. Cuando se usa como monoterapia en tumores T1 y T2 de lengua, se pueden conseguir tasas de supervivencia del 61 y $74 \%$, respectivamente (48), pero los resultados en los tumores mayores son pobres (49). Un mejor control de la enfermedad se obtiene cuando se combina la braquiterapia con irradiación de rayo externo a baja dosis, pero esto se asocia con necrosis de los tejidos blandos y osteorradionecrosis; un $2 \%$ requieren cirugía para tratar estas complicaciones (50).

La radioterapia se puede administrar como monoterapia o como complemento a la cirugía. Es tan eficaz como la cirugía para las pequeñas lesiones en la lengua y se puede escoger como tratamiento primario en aquellos casos en los que la cirugía pudiera resultar en discapacidad severa. La radioterapia normalmente se usa como complemento de la cirugía y en la actualidad se tiende a darla postoperatoriamente, habitualmente debido a una histología desfavorable. Muchos oncólogos recomiendan la radioterapia coadyuvante en tumores extensos si los márgenes quirúrgicos están próximos al tumor, o tras la disección ganglionar cuando existen múltiples nódulos positivos. En la práctica, se utiliza en las neoplasias en extensión regional (51). El argumento para el empleo de la radioterapia postoperatoria es que mejora el control local y, de esta forma, se mejora la calidad de vida del paciente, a pesar de que no mejore la supervivencia. Pero la radioterapia postoperatoria sobre la cavidad oral implica morbilidad. Las complicaciones más frecuentes son disfagia, ageusia, xerostomía, osteorradionecrosis y edema facial (52). Es la incidencia de estas complicaciones, frente a los potenciales beneficios, la que debe ser evaluada por cada centro a la hora de decantarse o no por su indicación.

Existe una correlación entre el control de la enfermedad mediante radioterapia y la morfología del tumor, con un mejor control local de la enfermedad conseguido en tumores superficiales y exofíticos, en comparación con los infiltrantes (53).
La radioterapia acelerada, continua e hiperfraccionada (CHART) se ha utilizado en un número limitado de centros de oncología para tratar tumores de cabeza y cuello. Los regímenes convencionales de radioterapia se basan en una única dosis diaria administrada cinco días a la semana. CHART se administra en dosis individuales más pequeñas, pero tres veces al día en un periodo continuado a lo largo de 12 días. El tratamiento sin intervalos es particularmente beneficioso en tumores con una rápida velocidad de multiplicación, ya que se reduce la posibilidad de reproducción tumoral durante las pausas en el tratamiento. Además, la radioterapia dada en múltiples pequeñas dosis puede causar a largo plazo daño a los tejidos normales. Se ha observado una mejora en la supervivencia y en el control local del tumor cuando se utiliza CHART en el tratamiento de carcinomas localmente avanzados de cabeza y cuello. Cuando se utiliza para tratar lesiones de la lengua, se observa una reacción membranosa en el dorso y en los laterales, que puede tardar varios meses en cicatrizar (54).

\section{Quimioterapia}

La quimioterapia convencional tiene un papel limitado en el manejo primario del carcinoma de la lengua, pero en ocasiones se considera como un coadyuvante cuando la enfermedad es avanzada. La quimioterapia se fundamenta en el uso de medicamentos contra el cáncer suministrados vía intravenosa o vía oral. Estos medicamentos ingresan en el torrente sanguíneo y pueden llegar al cáncer que se ha propagado a los órganos más allá de la cabeza y del cuello. Esta terapia se puede emplear en varias situaciones diferentes.

Se puede usar quimioterapia combinada con radioterapia, en lugar de cirugía, para controlar los tumores cancerosos más grandes que están confinados a la región de la cabeza y el cuello. Por lo general, no se usa la quimioterapia sin radiación para tratar estos tumores cancerosos.

En ocasiones, la quimioterapia se suministra (a veces con radiación) para reducir el cáncer antes de la cirugía. Esto se llama quimioterapia neoadyuvante o de inducción. En algunos casos, esto posibilita el uso de una cirugía menos radical y la extirpación de 
menos tejido. Si bien este tipo de tratamiento puede conducir a menos efectos secundarios graves a causa de la cirugía, no parece ayudar a que los pacientes vivan durante más tiempo.

La quimioterapia (por lo general, con radiación) se ha empleado también para reducir la gravedad de los síntomas del cáncer de cabeza y cuello en los casos en que el tumor es demasiado grande para ser extirpado por completo y la radioterapia por sí sola no lo ha podido controlar.

Los medicamentos de quimioterapia que se utilizan con mayor frecuencia para el cáncer de la cavidad oral y de la orofaringe son: Cisplatino, 5-fluorouracilo (5-FU), carboplatino, paclitaxel, docetaxel, metotrexato, ifosfamida, bleomicina.

\section{Nuevos enfoques en la quimioterapia}

Gran parte de la investigación se está enfocando en mejorar los resultados de la quimioterapia en las personas con este tipo de cáncer. Esto incluye encontrar el momento propicio para suministrar estos medicamentos, averiguar cuáles son las combinaciones de medicamentos que funcionan mejor, y determinar cómo usar mejor estos medicamentos con otras formas de tratamiento. También se están desarrollando nuevos medicamentos de quimioterapia que podrían ser más eficaces contra los tumores cancerosos avanzados de la cavidad oral y de la orofaringe. La combinación de docetaxel, cisplatino, y 5- fluorouracilo (TPF) suministrada antes de la radioterapia parece funcionar mejor que el cisplatino y el 5-fluorouracilo solo. Actualmente se está probando su función en combinación con la radiación y otros agentes quimioterapéuticos en estudios más amplios. En un enfoque más reciente para el cáncer de cabeza y cuello se inyecta el medicamento directamente en el tumor (quimioterapia intralesional). El éxito con este enfoque ha sido limitado en el pasado porque el medicamento tendía a propagarse rápidamente fuera de los tumores, a los tejidos cercanos y al resto del cuerpo. Los últimos avances en la preparación de la solución del medicamento de forma que ésta permanezca en el tumor (tal como una suspensión en gel) han renovado el interés en este tipo de tratamiento.

\section{Terapia dirigida}

Algunos medicamentos que se dirigen al receptor del factor de crecimiento epidérmico (EGFR) pueden ayudar a tratar los tipos de cáncer de la cavidad oral y de la orofaringe. Ya se aprobó el cetuximab (Erbitux) para el uso contra estos tipos de cáncer. Actualmente se están estudiando otros medicamentos incluyendo el erlotinib, panitumumab, lapatinib, y gefitinib. Ahora también se están estudiando medicamentos que bloquean el crecimiento de los vasos sanguíneos que los tumores necesitan para sobrevivir, tales como el bevacizumab y el sunitinib, para ser usados contra estos tipos de cáncer (55).

\section{Nuevos tratamientos}

Actualmente las investigaciones intentan desentrañar el mecanismo genético que origina el cáncer de lengua. Sabemos que la alteración del oncogén supresor que codifica para la proteína p53 está muy relacionado con la aparición de muchos de los carcinomas epidermoides de cabeza y cuello. Además están involucrados otros genes como el erb-b1 y el c-myc. Cada vez hay más evidencia de que las infecciones virales pueden tener un papel en el origen de un cáncer de lengua. Se están desarrollando nuevos tratamientos entre los que se encuentran varias terapias biológicas (por ejemplo, vacunas, antagonistas del receptor del factor de crecimiento, inhibidores de la cinasa dependientes de la ciclina, virus oncolíticos, y otros); hay algunos tipos de vacunas en etapas iniciales de investigación que están siendo estudiados como una forma de tratar a las personas con cáncer ayudando a su sistema inmunológico a reconocer y combatir las células cancerosas. Muchas de estas vacunas involucran a las células dendríticas, que son extraídas de la sangre del paciente y expuestas en el laboratorio a algo que las estimule para atacar las células del tumor. Luego, las células dendríticas son nuevamente inyectadas en el cuerpo, donde deberían inducir a otras células del sistema inmunológico a atacar el cáncer del paciente (56).

Por último, destacar la electroquimioterapia, mediante la cual se inyecta bleomicina intratumoral, para a continuación aplicar un tratamiento eléctrico de alto 
voltaje, lo cual induce a la rápida necrosis del tejido tumoral (57).

\section{BIBLIOGRAFÍA}

1. Horner MJ, Ries LAG, Krapcho M, Neyman N, Aminou R, Howlader $\mathrm{N}$, et al. SEER Cancer Statistics Review, 1975-2006, National Cancer Institute. Bethesda, MD.

2. Cancer Facts $\&$ Figures. Atlanta: American Cancer Society, 2011.

3. Parkin DM, Whelan SL, Ferlay J, et al. eds. Cancer incidence in five continents, vol. VII. IARC scientific publications No. 120. Lyon, International Agency for Research on Cancer, 1997.

4. Speight PM, Farthing PM, Bouquot JE. The pathology of oral cancer and precancer. Current Diagnostic Pathol 1996;3:165-76.

5. Warnakulasuriya S, Johnson NW, van der Waal I. Nomenclature and classification of potentially malignant disorders of the oral mucosa. J Oral Pathol Med 2007;36:575-80.

6. Lynch, Brightman G. Medicina bucal de Burket. 9a edn. México: McGraw-Hill Interamericana, 1996.

7. Scully C, Field JK, Tanzawa H. Genetic aberrations in oral or head and neck squamous cell carcinoma (SCCHN): 1. Carcinogen metabolism, DNA repair and cell cycle control. Oral Oncol 2000;36:256-63.

8. Braakhuis BJ, Tabor MP, Kummer JA, Leemans $\mathrm{CR}$, Brakenhoff $\mathrm{RH}$. A genetic explanation of Slaughter's concept of field cancerization: evidence and clinical implications. Cancer Res 2003;63:1727-30.

9. Macfarlane GJ, Zheng T, Marshall JR, Boffetta P, Niu S, Brasure J et al. Alcohol, tobacco, diet and the risk of oral cancer: a pooled analysis of three case-control studies. Eur J Cancer B Oral Oncol 1995;31B:181-7.
10. Nieto A, Ramos M. Rising tends in oral cancer mortality in Spain, 1975-94. J Oral Pathol Med 2002;31:147-52.

11. Zhang ZF, Morgenstern H, Spitz MR, Tashkin DP, Yu GP, Marshall JR et al. Marijuana use and increased risk of squamous cell carcinoma of the head and neck. Cancer Epidemiol Biomark Prev 1999;8:1071-8.

12. Marandas P, Marandas N. Situation actuelle des cancers des vois aéro-digestives supérieures en France et données épidemiologiques. In: Marandas P. Editor. Cancers des vois aérodigestives supérieures. Données actualles Issyles-Moulineaux: Masson, 2004.

13. Maier H, Sennewald E, Héller G, Weidauer H. Chronic alcohol consumption- the key risk factor for pharyngeal cancer.Otolaryngol head Neck Surg 1994;110:168-73.

14. Schwartz SM, Doody DR, Fitzgibbons ED, Ricks S, Porter PL, Chen C. Oral squamous cell cancer risk in relation to alcohol consumption and alcohol dehydrogenase-3 genotypes. Cancer Epidemiol Biomarkers Prev 2001;10:1137-44.

15. Franceschi S, Barra S, La Vecchia C, Bidoli E, Negri E, Talamini R. Risk factors for cancer of the tongue and the mouth. A case-control study from northern Italy. Cancer 1992; 70: 2227-33.

16. Carretero Peláez MA, Esparza Gómez GC, Figuero Ruiz E, Cerero Lapiedra R. Alcohol-containing mouthwashes and oral cancer. Critical analysis of literature. Med Oral 2004;9:120-3, 116-20.

17. Scully C. Oral cancer; the evidence for sexual transmission. Br Dent J 2005;199:203-7.

18. Mork J, Lie AK, Glattre E, Hallmans G, Jellum E, Koskela $\mathrm{P}$ et al. Human papillomavirus infection as a risk factor for squamous-cell carcinoma of the head and neck. N Engl J Med 2001;344:112531.

19. Llamas-Martínez S, Esparza-Gómez G, CampoTrapero J, Cancela- Rodríguez P, Bascones-Mar- 
tínez A, Moreno-López LA et al. Genotypic determination by PCR-RFLP of human papillomavirus in normal oral mucosa, oral leukoplakia and oral squamous cell carcinoma samples in Madrid (Spain). Anticancer Res 2008;28:3733-41.

20. Gillison ML, Koch WM, Shah KV. Human papillomavirus in head and neck squamous cell carcinoma: are some head and neck cancers a sexually transmitted disease? Curr Opin Oncol 1999;11:191-9.

21. Gillison ML, Koch WM, Capone RB, Spafford M, Westra WH, Wu L et al. Evidence for a causal association between human papillomavirus and a subset of head and neck cancers. J Natl Cancer Inst 2000;92:709-20.

22. Kantola S, Parikka M, Jokinen K, Hyrynkangs K, Soini Y, Alho OP et al. Prognostic factors in tongue cancer - relative importance of demographic, clinical and histopathological factors. Br J Cancer 2000;83:614-9.

23. Liang XH, Lewis J, Foote R, Smith D, Kademani D. Prevalence and significance of human papillomavirus in oral tongue cancer: the Mayo Clinic experience. J Oral Maxillofac Surg 2008; 66:1875-80.

24. Dahlgren L, Dahlstrand HM, Lindquist D, Hogmo A, Bjornestal L, Lindholm $\mathrm{J}$ et al. Human papillomavirus is more common in base of tongue than in mobile tongue cancer and is a favorable prognostic factor in base of tongue cancer patients. Int J Cancer 2004;112:1015-9.

25. Heck JE, Berthiller J, Vaccarella S, Winn DM, Smith EM, Shangina $O$ et al. Sexual behaviours and the risk of head and neck cancers: a pooled analysis in the International Head and Neck Cancer Epidemiology (INHANCE) consortium. Int J Epidemiol 2010;39:166-81.

26. D'Souza G, Agrawal Y, Halpern J, Bodison S, Gillison ML. Oral sexual behaviors associated with prevalent oral human papillomavirus infection. J Infect Dis 2009;199:1263-9.
27. Prince S, Bailey BM. Squamous carcinoma of the tongue: review. Br J Oral Maxillofac Surg 1999;37:164-74.

28. Gorsky M, Epstein JB, Oakley C, Le ND, Hay J, Stevenson-Moore P. Carcinoma of the tongue: a case series analysis of clinical presentation, risk factors, staging, and outcome. Oral Surg Oral Med Oral Pathol Oral Radiol Endod 2004;98:546-52.

29. Wu H, Ying H, Xi X, Shen N, Shu Y, Hoffman MR et al. Localization of the sentinel lymph node in tongue VX2 carcinoma via indirect CT lymphography combined with methylene blue dye injection. Acta Otolaryngol 2010;130:503-10.

30. Braams JW, Pruim J, Freling NJ, Nikkels PG, Roodenburg JL, Boering G et al. Detection of lymph node metastases of squamous-cell cancer of the head and neck with FDG-PET and MRI. J Nucl Med 1995;36:211-6.

31. Watkinson JC, Lazarus CR, Todd C, Maisey MN, Clarke SE. Metastatic squamous carcinoma in the neck: an anatomical and physiological study using CT and SPECT 99Tcm (V) DMSA. Br J Radiol 1991;64:909-14.

32. Levendag P, Teguh D, Heijmen B. Prognostic Factors. In: Halperin E, Perez C, Brady L, ed. Perez and Brady's Principles and practice of radiation oncology. Philadelphia: Lippincott Williams E Wilkins 2008:922.

33. Shah JP. Cervical lymph node metastases-diagnostic, therapeutic, and prognostic implications. Oncology (Williston Park) 1990;4:61-9; discussion 72,76 .

34. Shah JP, Andersen PE. Evolving role of modifications in neck dissection for oral squamous carcinoma. Br J Oral Maxillofac Surg 1995;33:3-8

35. Haddadin KJ, Soutar DS, Oliver RJ, Webster MH, Robertson AG, MacDonald DG. Improved survival for patients with clinically T1/T2, N0 tongue tumors undergoing a prophylactic neck dissection. Head Neck 1999 21:517-25. 
36. Andersen PE, Cambronero E, Shaha AR, Shah JP. The extent of neck disease after regional failure during observation of the NO neck. Am J Surg 1996;172:689-91.

37. Takagi $M$, Kayano T, Yamamoto $H$, Shibuya $H$, Hoshina M, Shioda S et al. Causes of oral tongue cancer treatment failures. Analysis of autopsy cases. Cancer 1992;69:1081-7.

38. Hu K, Harrison L, Culliney B. Cancer of the oropharynx. In: Harrison LB, Sessions RB, Hong WK, eds.: Head and Neck Cancer: A Multidisciplinary Approach. 2nd ed. Philadelphia Pa: Lippincott Williams \& Wilkins, 2004:306-51.

39. D'Souza G, Kreimer AR, Viscidi R, Pawlita M, Fakhry D, Koch WM et al. Case-control study of human papillomavirus and oropharyngeal cancer. N Engl J Med 2007;356:1944-56.

40. Tabor MP, Brakenhoff RH, Ruijter-Schippers HJ, Van Der Wal JE, Snow GB, Leemans CR et al. Multiple head and neck tumors frequently originate from a single preneoplastic lesion. Am J Pathol 2002;161:1051-60.

41. Woolgar JA. Histopathological prognosticators in oral and oropharyngeal squamous cell carcinoma. Oral Oncol 2006;42:229-39.

42. Finlay PM, Dawson F, Robertson AG, Soutar DS. An evaluation of functional outcome after surgery and radiotherapy for intraoral cancer. $\mathrm{Br} \mathrm{J}$ Oral Maxillofac Surg 1992;30:14-7.

43. Gil Z, Fliss DM. Contemporary management of head and neck cancers. Isr Med Assoc J 2009;11: 296-300.

44. Kraus DH, Vastola AP, Huvos AG, Spiro RH. Surgical management of squamous cell carcinoma of the base of the tongue. Am J Surg 1993; 166:384-8.

45. Vega C, León X, Cervelli D, Pons G, López S, Fernández $M$ et al. Total or subtotal glossectomy with microsurgical reconstruction: functional and oncological results. Microsurgery 2011;31:517-23.
46. Dziegielewski PT, Ho ML, Rieger J, Singh P, Langille M, Harris JR et al. Total glossectomy with laryngeal preservation and free flap reconstruction: objective functional outcomes and systematic review of the literature. Laryngoscope 2013;123: 140-5.

47. Mañon R, Myers J, Khuntia D, Harari P. Dental Care. In: Halperin E, Perez C, Brady L, ed. Perez and Brady's Principles and practice of radiation oncology. Philadelphia: Lippincott Williams E Wilkins 2008:906.

48. Henk JM. Treatment of oral cancer by interstitial irradiation using iridium-192. Br J Oral Maxillofac Surg 1992;30:355-9.

49. Podd TJ, Carton AT, Barrie R, Dawes PK, Roberts JT, Stassen LF et al. Treatment of oral cancers using iridium-192 interstitial irradiation. Br J Oral Maxillofac Surg 1994;32:207-13.

50. Harrison LB, Zelefsky MJ, Sessions RB, Fass DE, Armstrong JG, Pfister DG et al. Base-of-tongue cancer treated with external beam irradiation plus brachytherapy: oncologic and functional outcome. Radiology 1992;184:267-70.

51. Mendenhall W, Werning J, Pfister D. Principles of Treatment for Squamous Cell Carcinoma. General Principles for Selection of Treatment. In: DeVita V, Lawrence T, Rosenberg S, ed. Devita, Hellman $\mathcal{E}$ Rosenberg's Cancer: Principles $\mathcal{E}$ Practice of Oncology: Lippincott Williams $\mathcal{E}$ Wilkins 2008:813.

52. Guchelaar HJ, Vermes A, Meerwaldt JH. Radiation-induced xerostomia: pathophysiology, clinical course and supportive treatment. Support Care Cancer 1997;5:281-8.

53. Shibuya H, Hoshina M, Takeda M, Matsumoto S, Suzuki S, Okada N. Brachytherapy for stage I $\mathcal{E}$ II oral tongue cancer: an analysis of past cases focusing on control and complications. Int $\mathrm{J}$ Radiat Oncol Biol Phys 1993;26:51-8.

54. Dische S, Saunders MI. The rationale for continuous, hyperfractionated, accelerated 
radiotherapy (CHART). Int J Radiat Oncol Biol Phys 1990;19:1317-20.

55. Last Medical Review. 4/11/2011. Copyright American Cancer Society.

56. De Costa AM, Justis DN, Schuyler CA, Young MR. Administration of a vaccine composed of dendritic cells pulsed with premalignant oral lesion lysate to mice bearing carcinogen-induced premalignant oral lesions stimulates a protective immune response. Int Immunopharmacol 2012;13:322-30.

57. Omura S, Tsuyuki Y, Ohta S, Li X, Bukawa H, Fujita K. Rapid tumor necrosis induced by electrochemotherapy with intratumoral injection of bleomycin in a hamster tongue cancer model.Int J Oral Maxillofac Surg 2000;29:11925.

\section{CORRESPONDENCIA}

Ana Isabel García Kass Dpto. Estomatología III. Facultad de Odontología. Universidad Complutense de Madrid Plaza Ramón y Cajal, s/n 28040 Madrid

Correo electrónico: garciakass@yahoo.es 